\title{
Dipole-dipole shift of quantum emitters coupled to surface plasmons of a nanowire
}

\author{
David Dzsotjan, ${ }^{1,2}$ Jürgen Kästel, ${ }^{1, *}$ and Michael Fleischhauer ${ }^{1}$ \\ ${ }^{1}$ Department of Physics and Research Center OPTIMAS, University of Kaiserslautern, D-67663 Kaiserslautern, Germany \\ ${ }^{2}$ Research Institute for Particle and Nuclear Physics, H-1525 Budapest, Hungary
}

(Received 10 May 2011; published 5 August 2011)

\begin{abstract}
Placing quantum emitters close to a metallic nanowire, an effective interaction can be achieved over distances large compared to the resonance wavelength due to the strong coupling between emitters and the surface plasmon modes of the wire. This leads to modified collective decay rates, as well as to Lamb and dipole-dipole shifts. In this paper, we present a general method for calculating these level shifts, which we subsequently apply to the case of a pair of atoms coupled to the guided modes of a nanowire.
\end{abstract}

DOI: 10.1103/PhysRevB.84.075419

PACS number(s): 42.50.Nn, 42.50.Ct, 78.67.Uh

\section{INTRODUCTION}

Coupling quantum systems to a common reservoir results in an effective interaction between these systems. In the case of spontaneous emission of light from quantum emitters, this manifests itself in phenomena such as superradiance and dipole-dipole interactions. In free space, these effects quickly disappear as soon as the average distance between the emitters exceeds the resonant wavelength. The situation changes dramatically, however, if the most dominant reservoir modes are reduced to one or zero spatial dimensions as in the case of a nanowire or a single-mode resonator. Here, interactions over large distances can emerge. Both the collective decay rates as well as the effective interaction Hamiltonian are fully determined by the dyadic Green's function of the electromagnetic field characterizing the response of the tailored reservoir. Calculating the collective decay rates requires one to determine the Green's tensor at a given frequency, typically the resonance frequency of the involved emitters. However, in order to determine the level shifts, one must perform a principal value integral over the whole positive frequency spectrum, which raises serious additional difficulties.

In this paper, we present a method to simplify the calculation of the Lamb and dipole-dipole shifts. One of the key steps is to introduce imaginary frequencies and extend the integrals over frequency into the complex plane. There are also other situations when introducing imaginary frequencies and using complex frequency integrals helps to simplify the original problem, for example, in the case of calculating Casimir-Polder potentials and investigating van der Waals interactions (Refs. 1-3 and references therein). We also introduce a special Kramers-Kronig relation which, combined with the aforementioned integral extension, allows us to transform the original expression for the level shifts into a much more convenient form.

We apply the method to a particular example where a pair of quantum emitters are coupled to the surface plasmon modes of a nanowire. This system is interesting because it enables one to attain strong atom-field coupling and single-site addressability at the same time. Because of the small transverse mode area of the plasmons of a metallic cylinder with a subwavelength radius, a strong Purcell effect arises between the plasmons and a single emitter placed close to the wire. ${ }^{4-6}$ The effect of strong coupling to the plasmon modes of a waveguide has been studied for various specific scenarios. ${ }^{7,8}$ The system of a single emitter coupled to a wire has been proposed as an efficient single-photon generator, ${ }^{4}$ as well as a single-photon transistor, ${ }^{9}$ and the coupling has been experimentally demonstrated. ${ }^{10}$ Having a pair of emitters coupled to the guided modes, we derived an interemitter distance dependent superradiance effect in Ref. 6. Here we calculate the dipole-dipole level shifts of the two-atom system.

\section{GENERAL METHOD}

Let us consider a system of $N$ two-level quantum emitters, characterized by the lowering and raising operators $\hat{\sigma}_{j}$ and $\hat{\sigma}_{j}^{\dagger}$ coupled to a common electromagnetic reservoir. Under conditions that permit the dipole, rotating wave, and Markov approximation tracing out the reservoir modes leads to a master equation for the atoms,

$$
\begin{aligned}
\dot{\hat{\rho}}= & i \sum_{m, n=1}^{N} \delta \omega_{m n}\left[\hat{\sigma}_{m}^{\dagger} \hat{\sigma}_{n}, \hat{\rho}\right] \\
& -\sum_{m, n=1}^{N} \frac{\Gamma_{m n}}{2}\left(\hat{\sigma}_{m}^{\dagger} \hat{\sigma}_{n} \hat{\rho}+\hat{\rho} \hat{\sigma}_{m}^{\dagger} \hat{\sigma}_{n}-2 \hat{\sigma}_{n} \hat{\rho} \hat{\sigma}_{m}^{\dagger}\right)
\end{aligned}
$$

The first, Hermitian term of the right-hand side (RHS) contains the $\delta \omega_{n n}$ Lamb shifts and $\delta \omega_{m \neq n}$ dipole-dipole shifts. In the dissipative term, we find the single-atom decay rates $\Gamma_{n n}$ and the interatomic decay couplings $\Gamma_{m \neq n}$. Their explicit form is

$$
\begin{gathered}
\Gamma_{m n}\left(\omega_{A}\right)=\frac{2 \omega_{A}^{2} d_{m_{i}} d_{n_{j}}}{\hbar \epsilon_{0} c^{2}} \operatorname{Im}\left[G_{i j}\left(\vec{r}_{m}, \vec{r}_{n}, \omega_{A}\right)\right], \\
\delta \omega_{m n}\left(\omega_{A}\right)=\frac{d_{m_{i}} d_{n_{j}}}{\hbar \epsilon_{0} \pi} \mathbb{P} \int_{0}^{\infty} d \omega \frac{\omega^{2}}{c^{2}} \frac{\operatorname{Im}\left[G_{i j}\left(\vec{r}_{m}, \vec{r}_{n}, \omega\right)\right]}{\omega-\omega_{A}} .
\end{gathered}
$$

Here, $G_{i j}\left(\vec{r}_{m}, \vec{r}_{n}, \omega\right)$ is the $\{i, j\}$ component of the Green's tensor for the electromagnetic field including the interaction with a passive medium such as a nanowire, evaluated at frequency $\omega$ and at positions $\vec{r}_{m}$ and $\vec{r}_{n}$. It fulfills the MaxwellHelmholtz wave equation,

$$
\left[\nabla \times \frac{1}{\mu(\vec{r}, \omega)} \nabla \times-\frac{\omega^{2}}{c^{2}} \epsilon(\vec{r}, \omega)\right] \overline{\bar{G}}\left(\vec{r}, \vec{r}^{\prime}, \omega\right)=\overline{\bar{I}} \delta\left(\vec{r}-\vec{r}^{\prime}\right),
$$

with the proper boundary conditions. $\epsilon(\vec{r}, \omega)$ and $\mu(\vec{r}, \omega)$ are the relative electric permittivity and magnetic permeability, respectively. In the case of transition frequencies $\omega_{A}$ for which 
the rotating-wave approximation is valid and which are far from the ultraviolet domain, we can use the full (vacuum plus material part) Green's tensor for calculating $\Gamma_{m n}$. For calculating $\delta \omega_{m n}$, one has to perform however an integral over the whole spectrum. Since the atom-field coupling is treated in a nonrelativistic way, this approach does not take into account the relativistic high-frequency contributions correctly. A well-known consequence of this is that the vacuum level shifts (Lamb shift) obtained from Eq. (3) are incorrect. Here, a rather involved calculation based on relativistic quantum field theory is required, taking into account all possible transitions of the emitter and including proper renormalizations. However, if we are interested only in the changes produced by the presence of a medium, this problem can be circumvented. The medium tailors the reservoir modes only within a certain frequency range and becomes transparent in the high-frequency domain. Thus calculating the effects relative to the case of free-space vacuum, i.e., using $\overline{\bar{G}}^{\text {med }}=\overline{\bar{G}}-\overline{\bar{G}}^{\text {vac }}$ instead of the full $\overline{\bar{G}}$, the above equations give correct expressions also for the Lamb or dipole-dipole shifts relative to vacuum. In other words, calculating only the material-induced level shift introduces an automatic renormalization and allows us to get rid of the ultraviolet divergences.

Deriving Eqs. (2) and (3), we also used a Markov approximation. This is possible as long as the calculated decay rates $\Gamma_{m n}(\omega)$ and shifts $\delta \omega_{m n}(\omega)$ depend only slowly on frequency $\omega$, i.e., do not change appreciably over frequency ranges of the order of $\Gamma_{m n}$ and $\delta \omega_{m n}$. It should be kept in mind that even if the spectral response of the medium is flat, retardation effects can cause a spectral dispersion of the Green's tensor at two different positions $\vec{r}_{m}$ and $\vec{r}_{n}$ with a characteristic width given by $c /\left|\vec{r}_{m}-\vec{r}_{n}\right|{ }^{11}$

Even though ultraviolet divergencies are eliminated in Eq. (3) by considering only the changes due to the medium, the expression still contains an integral that is rather difficult to calculate. In the following, we will give a general method for simplifying this expression. For this we shall use a generalized Kramers-Kronig relation.

\section{A. Kramers-Kronig relations for the Green's tensor}

The full Green's tensor $\overline{\bar{G}}(\omega)$ does not have any poles on the upper complex half plane because of causality. Thus KramersKronig relations (see Appendix A) apply, e.g.,

$$
\operatorname{Re}\left\{\overline{\bar{G}}\left(\omega_{A}\right)\right\}=\frac{1}{\pi} \mathbb{P} \int_{-\infty}^{\infty} d \omega \frac{\operatorname{Im}\{\overline{\bar{G}}(\omega)\}}{\omega-\omega_{A}}
$$

Because the Green's function inherits the symmetry $\overline{\bar{G}}\left(-\omega^{*}\right)=\overline{\bar{G}}^{*}(\omega)$ from $\epsilon(\omega)$, Eq. (5) can also be written in the form

$$
\operatorname{Re}\left\{\overline{\bar{G}}\left(\omega_{A}\right)\right\}=\frac{2}{\pi} \mathbb{P} \int_{0}^{\infty} d \omega \frac{\omega \operatorname{Im}\{\overline{\bar{G}}(\omega)\}}{\omega^{2}-\omega_{A}^{2}} .
$$

An important step in the derivation of the Kramers-Kronig relation is the integration over the semicircle ( $\curvearrowleft$ contribution) in the complex upper half plane. As this integration is done for large $|\omega|$, using the vacuum Green's tensor, ${ }^{12}$

$$
\begin{aligned}
\overline{\bar{G}}^{\mathrm{vac}}(\vec{r}, \omega)= & \frac{e^{i \frac{\omega}{c} r}}{4 \pi r}\left[\left(1-\frac{1}{i \frac{\omega}{c} r}-\frac{1}{\frac{\omega^{2}}{c^{2}} r^{2}}\right) \mathbb{1}\right. \\
& \left.+\left(-1+\frac{3}{i \frac{\omega}{c} r}+\frac{3}{\frac{\omega^{2}}{c^{2}} r^{2}}\right) \hat{r} \otimes \hat{r}\right]+\frac{\delta(\vec{r})}{3 \frac{\omega^{2}}{c^{2}}} \mathbb{1}
\end{aligned}
$$

is a good approximation. At large $|\omega|$, it goes as

$$
\lim _{|\omega| \rightarrow \infty} \overline{\bar{G}}^{\mathrm{vac}}(\vec{r}, \omega)=\frac{e^{i \frac{\omega}{c} r}}{4 \pi r}(\mathbb{1}-\hat{r} \otimes \hat{r}) .
$$

To perform the $\curvearrowleft$ integral, we use $\omega=|\omega| e^{i \alpha}$ and approach the two points where the $\curvearrowleft$ part joins the real axis $(\delta \leqslant \alpha \leqslant$ $\pi-\delta)$. We then find

$$
\begin{aligned}
I & =|\omega| \int_{\curvearrowleft} d\left(e^{i \alpha}\right) e^{i \frac{r}{c}|\omega| e^{i \alpha}} \\
& =|\omega| \int_{1+i \delta}^{-1+i \delta} d\left(e^{i \alpha}\right) e^{i \frac{r}{c}|\omega| e^{i \alpha}} \\
& =-\frac{2 c}{r} \sin \left(\frac{|\omega|}{c} r\right) e^{-\frac{|\omega|}{c} r \delta} .
\end{aligned}
$$

Taking $|\omega|$ to infinity and $\delta$ to zero, we get

$$
\lim _{\delta \rightarrow 0} \lim _{|\omega| \rightarrow \infty} I=0 .
$$

Because the integrand on any point of the $\curvearrowleft$ contour part goes exponentially fast to zero, the integral will vanish on this part of the contour even if we multiply the integrand with a polynomial of $\omega$. In particular, we find that the variant of the Kramers-Kronig relation

$$
\frac{\omega_{A}^{2}}{c^{2}} \operatorname{Re}\left\{\overline{\bar{G}}\left(\omega_{A}\right)\right\}=\frac{2}{\pi} \mathbb{P} \int_{0}^{\infty} d \omega \frac{\omega^{2}}{c^{2}} \frac{\omega \operatorname{Im}\{\overline{\bar{G}}(\omega)\}}{\omega^{2}-\omega_{A}^{2}}
$$

holds as well. For the reasons stated above, Eqs. (5) and (11)_being true for $\overline{\bar{G}}$ and $\overline{\bar{G}}^{\text {vac }}$ _are valid for the material contribution $\overline{\bar{G}}^{\text {med }}$ also.

\section{B. Medium contribution to the Lamb and dipole-dipole shift}

We can rewrite the principal value integral in Eq. (3) as

$$
I_{1}=\mathbb{P} \int_{0}^{\infty} d \omega \frac{\omega^{2}}{c^{2}} \frac{\operatorname{Im}\left\{G_{i j}^{\mathrm{med}}\left(\vec{r}_{m}, \vec{r}_{n}, \omega\right)\right\}}{\omega^{2}-\omega_{A}^{2}}\left(\omega+\omega_{A}\right),
$$

where we replaced the full $\overline{\bar{G}}$ by $\overline{\bar{G}}^{\text {med }}=\overline{\bar{G}}-\overline{\bar{G}}^{\text {vac }}$, i.e., the contribution of the medium. We can now substitute the variant of the Kramers-Kronig relation (11) into Eq. (12), which yields

$$
\begin{aligned}
I_{1}= & \frac{\pi}{2} \frac{\omega_{A}^{2}}{c^{2}} \operatorname{Re}\left\{G_{i j}^{\mathrm{med}}\left(\omega_{A}\right)\right\} \\
& +\operatorname{Im}\left\{\mathbb{P} \int_{0}^{\infty} d \omega \frac{\omega^{2}}{c^{2}} G_{i j}^{\mathrm{med}}(\omega) \frac{\omega_{A}}{\omega^{2}-\omega_{A}^{2}}\right\} .
\end{aligned}
$$

Now we will try to eliminate the principal value from the second term of Eq. (13). To do this, we will have to transfer the integral from the real axis to the imaginary axis. This, however, shall have its advantages because the Green's tensor behaves much more smoothly for purely imaginary 
frequencies: oscillations become exponentially decreasing functions.

Resolving the principal value,

$$
\begin{aligned}
\mathbb{P}\left(\frac{\omega_{A}}{\omega^{2}-\omega_{A}^{2}}\right)= & \frac{1}{2}\left(\frac{1}{\omega-\omega_{A}-i \delta}-i \pi \delta\left(\omega-\omega_{A}\right)\right. \\
& \left.-\frac{1}{\omega+\omega_{A}-i \delta}+i \pi \delta\left(\omega+\omega_{A}\right)\right),
\end{aligned}
$$

the second term in Eq. (13) assumes the form

$$
\begin{aligned}
I_{2}= & -\frac{\pi}{2} \operatorname{Im}\left\{i \frac{\omega_{A}^{2}}{c^{2}} G_{i j}^{\mathrm{med}}\left(\omega_{A}\right)\right\}+\frac{1}{2} \operatorname{Im}\left\{\int_{0}^{\infty} d \omega \frac{\omega^{2}}{c^{2}} G_{i j}^{\mathrm{med}}(\omega)\right. \\
& \left.\times\left(\frac{1}{\omega-\omega_{A}-i \delta}-\frac{1}{\omega+\omega_{A}-i \delta}\right)\right\} .
\end{aligned}
$$

Because the integral goes from zero to $\infty$ on the real axis, we can create a closed contour in the upper right quarter of the complex plane. The integral over the curved part $(\mathcal{C})$ of the contour will again disappear, so we can write

$$
\int_{0}^{\infty} \rightarrow \oint-\int_{\mathcal{C}}-\int_{i \infty}^{0}=\oint-\int_{i \infty}^{0}
$$

Using $\omega=i \kappa$ on the imaginary axis, we get

$$
\begin{aligned}
I_{2}= & \frac{\pi}{2} \frac{\omega_{A}^{2}}{c^{2}} \operatorname{Re}\left\{G_{i j}^{\mathrm{med}}\left(\omega_{A}\right)\right\}-\operatorname{Im}\left\{\int_{0}^{\infty} d \kappa \frac{\kappa^{2}}{c^{2}} \frac{i G_{i j}^{\mathrm{med}}(i \kappa)}{2}\right. \\
& \left.\times\left(\frac{1}{i \kappa-\omega_{A}}-\frac{1}{i \kappa+\omega_{A}}\right)\right\} .
\end{aligned}
$$

Substituting Eq. (17) into Eq. (13) and then that into Eq. (3), we get for the Lamb and dipole-dipole shift

$$
\begin{aligned}
\delta \omega_{m n}\left(\omega_{A}\right)= & \frac{d_{m_{i}} d_{n_{j}}}{\hbar \pi \epsilon_{0}}\left[\pi \frac{\omega_{A}^{2}}{c^{2}} \operatorname{Re}\left\{G_{i j}^{\mathrm{med}}\left(\vec{r}_{m}, \vec{r}_{n}, \omega_{A}\right)\right\}\right. \\
& \left.+\int_{0}^{\infty} d \kappa \frac{\kappa^{2}}{c^{2}} \operatorname{Re}\left\{G_{i j}^{\mathrm{med}}\left(\vec{r}_{m}, \vec{r}_{n}, i \kappa\right)\right\} \frac{\omega_{A}}{\kappa^{2}+\omega_{A}^{2}}\right] .
\end{aligned}
$$

In this form we no longer have to worry about the principal value. As an additional benefit, transferring the integration to the imaginary axis makes the Green's tensor much better behaved (exponential decay instead of oscillations), which is very useful when calculating the shift by numerical means.

\section{Origin of the integral term}

The expression for the dipole-dipole shift found in the literature (for example, Refs. 13-15) usually involves only the first term of the right-hand side of Eq. (18) or, equivalently, the shift is proportional to the real part of the electric field at the atomic frequency. This is, however, an approximation that relies on the assumption that only frequencies around $\omega_{A}$ contribute to the dipole-dipole shift considerably. We can obtain this result if we only keep the first two terms in Eq. (14), arguing that the other terms have their greatest contribution at around $\omega=-\omega_{A}$, which is not contained by the region of integration in Eq. (12). However, there is a fundamental reason why, in general, the integral term in Eq. (18) must be present. Starting from Eq. (13), we can write

$$
\begin{aligned}
& \mathbb{P} \int_{0}^{\infty} d \omega \frac{\omega^{2}}{c^{2}} \frac{\operatorname{Im}\left\{G_{i j}^{\mathrm{med}}(\omega)\right\}}{\omega-\omega_{A}} \\
& \quad=\frac{\pi}{2} \frac{\omega_{A}^{2}}{c^{2}} \operatorname{Re}\left\{G_{i j}^{\mathrm{med}}\left(\omega_{A}\right)\right\}+\omega_{A} \mathbb{P} \int_{0}^{\infty} d \omega \frac{\omega^{2}}{c^{2}} \frac{\operatorname{Im}\left\{G_{i j}^{\mathrm{med}}(\omega)\right\}}{\omega^{2}-\omega_{A}^{2}} .
\end{aligned}
$$

Resolving the integral term on the RHS of Eq. (19), we get two terms, one of which is identical to the one on the LHS of the equation. Thus one obtains

$$
\begin{aligned}
& \mathbb{P} \int_{0}^{\infty} d \omega \frac{\omega^{2}}{c^{2}} \frac{\operatorname{Im}\left\{G_{i j}^{\mathrm{med}}(\omega)\right\}}{\omega-\omega_{A}} \\
& =\pi \frac{\omega_{A}^{2}}{c^{2}} \operatorname{Re}\left\{G_{i j}^{\mathrm{med}}\left(\omega_{A}\right)\right\}-\mathbb{P} \int_{0}^{\infty} d \omega \frac{\omega^{2}}{c^{2}} \frac{\operatorname{Im}\left\{G_{i j}^{\mathrm{med}}(\omega)\right\}}{\omega+\omega_{A}}
\end{aligned}
$$

Changing the integration variable on the RHS of Eq. (20) to $\tilde{\omega}=-\omega$ and using the fact that $\operatorname{Im}\left\{G_{i j}(\omega)\right\}$ is an odd function of $\omega$, we get

$$
\begin{aligned}
\mathbb{P} \int_{0}^{\infty} d \omega \frac{\omega^{2}}{c^{2}} \frac{\operatorname{Im}\left\{G_{i j}^{\mathrm{med}}(\omega)\right\}}{\omega-\omega_{A}}= & \pi \frac{\omega_{A}^{2}}{c^{2}} \operatorname{Re}\left\{G_{i j}^{\mathrm{med}}\left(\omega_{A}\right)\right\} \\
& -\mathbb{P} \int_{-\infty}^{0} d \tilde{\omega} \frac{\tilde{\omega}^{2} \operatorname{Im}\left\{G_{i j}^{\mathrm{med}}(\tilde{\omega})\right\}}{c^{2}}
\end{aligned}
$$

which, rearranged, gives us the variant of the Kramers-Kronig relation (11)

$$
\mathbb{P} \int_{-\infty}^{\infty} d \omega \frac{\omega^{2}}{c^{2}} \frac{\operatorname{Im}\left\{G_{i j}^{\mathrm{med}}(\omega)\right\}}{\omega-\omega_{A}}=\pi \frac{\omega_{A}^{2}}{c^{2}} \operatorname{Re}\left\{G_{i j}^{\mathrm{med}}\left(\omega_{A}\right)\right\} .
$$

Thus one sees that the term discussed in Eq. (19) is necessary in order to fulfill the Kramers-Kronig relations. Note that the integral term on the RHS of Eq. (20) can be transformed into the one we got in Eq. (18), making use of the well-known property of $\overline{\bar{G}}\left(\vec{r}_{1}, \vec{r}_{2}, \omega\right)$ to be real valued for purely imaginary frequencies.

As we will see later on for the setup discussed here and similar situations, the second term in Eq. (19) is indeed negligible for emitter separations much larger than the characteristic length (i.e., the wavelength). However, when the emitters get close enough, the static $(\omega=0)$ contribution in $\delta \omega_{12}$ increases substantially and we cannot neglect the integral term any more. Also there may be situations, such as the coupling of quantum emitters over macroscopic distances through a negative-index material, where this simple rule may not hold.

\section{PAIR OF EMITTERS NEAR A NANOWIRE}

In the following, we will apply the method introduced above to a particular example. From the interaction of two emitters, each coupled to the basic surface-plasmon (SP) mode of a metallic wire of subwavelength radius, emerges a Dicke superradiance effect that depends on the interemitter distance. ${ }^{6}$ Expressing the states of the atomic system in the well-known Dicke basis, as seen in Fig. 1, the full collective atomic decay 


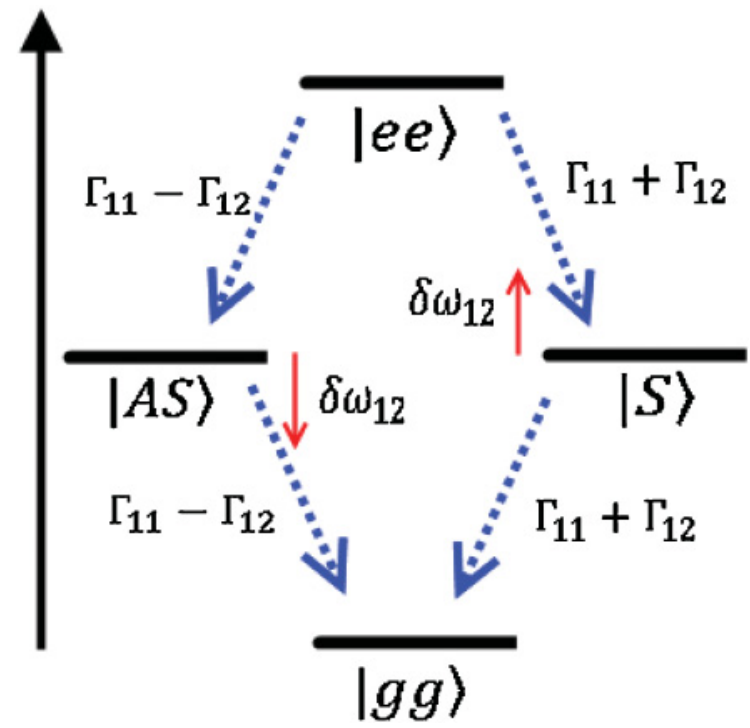

FIG. 1. (Color online) Collective atomic decays and dipole-dipole shifts of a pair of interacting two-level atoms, described in the Dicke basis. States $|e e\rangle$ and $|g g\rangle$ mean both atoms are in the excited and in the ground states, respectively. The symmetrized and antisymmetrized single-excitation states are defined as $|S\rangle=$ $1 / \sqrt{2}(|e g\rangle+|g e\rangle)$ and $|A S\rangle=1 / \sqrt{2}(|e g\rangle-|g e\rangle)$. If there is a strong effective coupling (high $\Gamma_{12}$ ) between the atoms, the difference between the collective decay rates becomes large, leading to a superradiance phenomenon.

rates are $\Gamma_{11} \pm \Gamma_{12}$. This means that the effective interaction between the atoms modifies the original single-atom decay rate $\Gamma_{11}$ by the coupling $\Gamma_{12}$ and the singly excited levels get $\mathrm{a} \pm \delta \omega_{12}$ interaction-induced dipole-dipole shift [see Eqs. (2) and (3)].

Trying to calculate Eq. (3) directly introduces difficulties, since one has to deal with a principal value integral. This is especially a problem if we have to perform the integral numerically (which is usually the case by nontrivial geometries), because we have to know the behavior of the $\overline{\bar{G}}^{\text {med }}(\omega)$ around $\omega=\omega_{A}$. As described in Ref. $6, \overline{\bar{G}}^{\text {med }}(\omega)=$ $\int_{0}^{\infty} d k_{z} \tilde{\bar{G}}^{\text {med }}\left(\omega ; k_{z}\right)$, which in this case is the scattered part of the Green's tensor. Although we know the analytic form of $\tilde{\overline{\bar{G}}}^{\text {med }}\left(k_{z} ; \omega\right)$, it is a rather complicated function and we cannot analytically integrate it. Using the method described in the previous section, however, circumvents these difficulties and allows us to perform the much simpler integration in Eq. (18) where, in addition, we have to substitute purely imaginary frequencies into the Green's tensor, by which we get a more well-behaved function.

We calculated the dipole-dipole shift resulting from the presence of the wire using the full scattered Green's tensor. We compared it to an analytic approximation used by Ref. 14, based on a single-plasmon resonance model, a derivation of which is given in Appendix B:

$$
\delta \omega_{12}^{\mathrm{appr}}=-\frac{2 \pi d^{2} \omega_{A}^{2}}{\hbar \epsilon_{0} c^{2}} A\left(\omega_{A}\right) \gamma\left(\omega_{A}\right) e^{-\gamma \Delta z} \sin \left(k_{z}^{\mathrm{pl}} \Delta z\right) .
$$

Here $A$ and $\gamma$ are the amplitude and width of the Lorentzian fit to the plasmonic resonances (see Appendix B) and

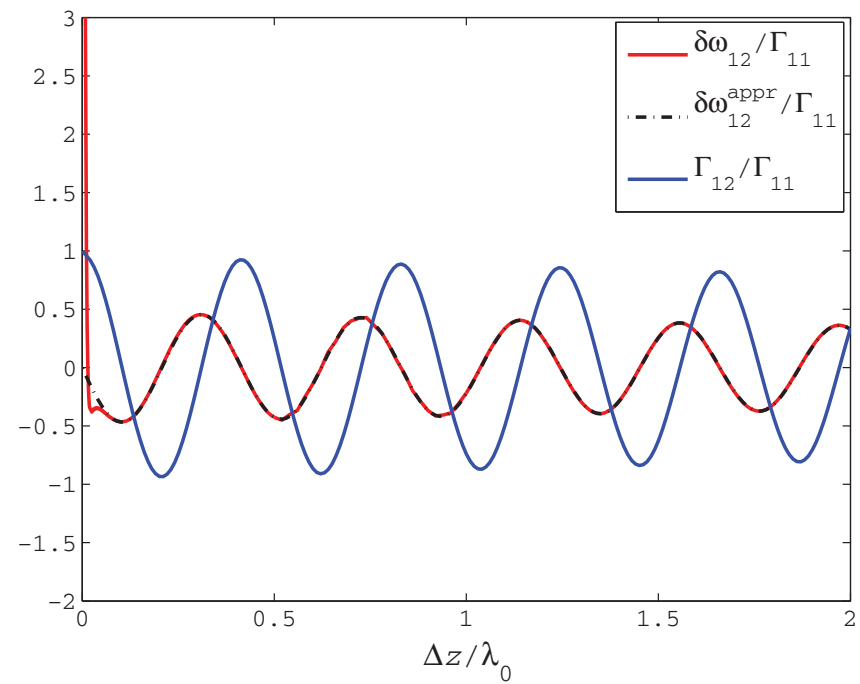

FIG. 2. (Color online) Dipole-dipole shift $\delta \omega_{12}$ due to the presence of the wire and the $\Gamma_{12}$ coupling between the atoms, scaled by the total single atom decay rate $\Gamma_{11}$, as a function of interatomic distance $\Delta z$ in units of the vacuum radiation wavelength $\lambda_{0} . \delta \omega_{12}^{\text {appr }}$ is a good analytic approximation of $\delta_{12}$, for distances comparable to $\lambda_{0}$ and higher. Here, $\delta \omega_{12} / \Gamma_{11}$ shows an oscillating behavior shifted by $\pi / 2$ with respect to $\Gamma_{12} / \Gamma_{11}$. For interemitter distances comparable to the wire radius, there is a substantial increase in $\delta \omega_{12}$ and it begins to strongly deviate from $\delta \omega_{12}^{\text {appr }}$.

$k_{z}^{\mathrm{pl}}$ is the longitudinal component of the wave vector of the plasmon mode. Figure 2 shows the results of the calculations. For interemitter distances larger than the vacuum radiaton wavelength, $\delta \omega_{12}^{\text {appr }}$ is a good approximation to the exact wire-induced dipole-dipole shift. It only deviates from the sinusoidal behavior when the interemitter distance becomes comparable to the wire radius: in this case, the atoms begin to be affected by the three-dimensional nature of the wire and the quasi-1D coupling approximation (i.e., $\delta \omega_{12}^{\mathrm{appr}}$ ) is no longer valid. However, because the wire is quite thin, this typically happens at distances well below the vacuum radiation wavelength, which means that in this regime the emitters are already strongly interacting through the vacuum as well. So we can safely say that the analytic approximation works well if the interemitter distance is above the vacuum radiation wavelength of the emitters. In the regime where the exact calculations are well approximated by $\delta \omega_{12}^{\mathrm{appr}}, \delta \omega_{12} / \Gamma_{11}$ oscillates with the same period as $\Gamma_{12} / \Gamma_{11}$ only with an additional $\pi / 2$ relative phase shift. ${ }^{14}$ This means that for interemitter distances yielding extrema for $\Gamma_{12} / \Gamma_{11}$, i.e., where the symmetric or antisymmetric transition is superradiant, $|S\rangle$ and $|A S\rangle$ are degenerate. On the other hand, when $\Gamma_{12} / \Gamma_{11}=0$, this degeneracy is lifted by $\left|\delta \omega_{12}\right|$ being maximal. The extrema of $\left|\delta \omega_{12}\right|$ are $0.5 \Gamma_{11}$ at most. The decay of the amplitude of the oscillations for both $\delta \omega_{12} / \Gamma_{11}$ and $\Gamma_{12} / \Gamma_{11}$ is caused by ohmic losses in the metal, represented by $\gamma$. Thus the interaction always makes a distinction between the symmetric and the antisymmetric transition: either by the different decay rates or by the lifted degeneracy of $|S\rangle$ and $|A S\rangle$.

As seen in Fig. 3, the closer the emitters are to each other, the more substantial the integral term of Eq. (18) becomes. This is in accordance with the arguments made in Sec. II C, 


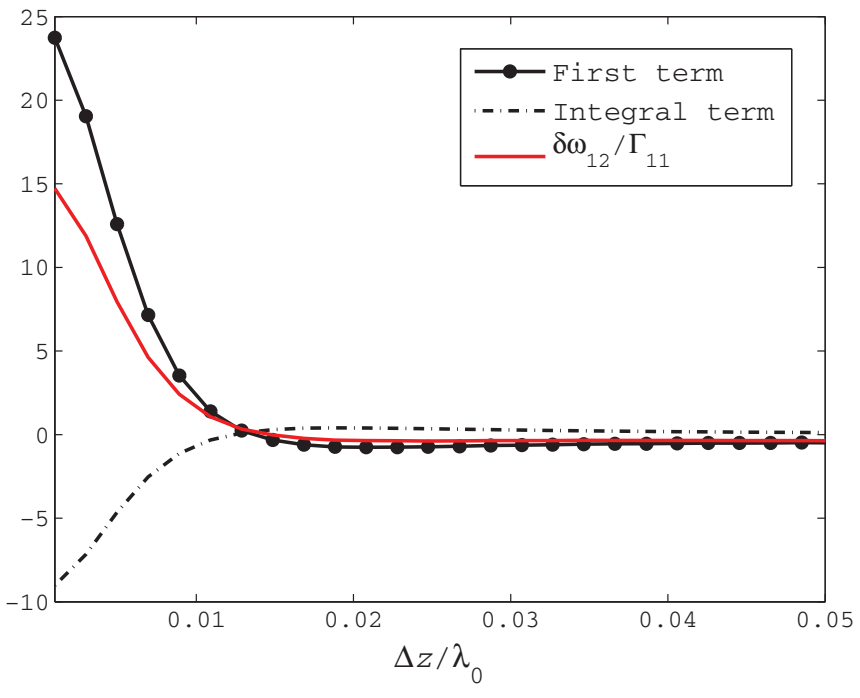

FIG. 3. (Color online) First term and the second, integral term of the dipole-dipole shift, as seen in Eq. (18) and the actual wire-induced dipole-dipole shift $\delta \omega_{12}$, scaled by the total single-emitter decay rate $\Gamma_{11}$. Because of the increasing static $(\omega=0)$ contributions in the Green's tensor, for small interemitter distances, the integral term becomes comparable to the first term, becoming no longer negligible.

namely that the decrease of the interemitter distance enhances the static contribution of the Green's tensor. For small enough distances, the integral term is not negligible any more.

\section{SUMMARY}

In the present paper, we discussed the effects of a tailored reservoir on the Lamb and dipole-dipole shifts of quantum emitters coupled to a common radiative reservoir. By considering only shifts relative to those in vacuum, all complications arising from ultraviolet divergencies and offresonant contributions from other transitions were eliminated, reducing the problem to the calculation of an integral of the electromagnetic Green tensor over all frequencies. We presented a method that greatly simplifies this calculation by transforming the original expression containing a principal value integral over frequency into an ordinary integration over the imaginary axis. The method does not imply any specific configuration or system, so it can be used in a wide variety of problems where level shifts due to dipole-dipole interaction have to be calculated. We discuss the appearance and importance of an integral term in the derived expression that sweeps across purely imaginary frequencies and is usually neglected in the literature. We apply the method for calculating the dipole-dipole shift of a pair of atoms coupled to the guided surface plasmon modes of a metallic nanowire and compare it to a quasi-1D analytic approximation. The results show that, for interemitter distances comparable to the wire radius, $\delta \omega_{12}$ becomes considerably larger than the single-atom decay rate $\left(\Gamma_{11}\right)$ and the approximation doesn't hold. However, for larger distances, the shift is very well approximated by the analytic expression, and $\delta \omega_{12} / \Gamma_{11}$ shows an oscillatory behavior having roughly half the amplitude and the same period as $\Gamma_{12} / \Gamma_{11}$, as well as an additional phase shift of $\pi / 2$. Thus the interaction always makes a distinction between the symmetric and antisymmetric transition of the two-atom system, either by the modified collective decay rates (superradiance) or the lifted degeneracy of $|S\rangle$ and $|A S\rangle$. We also take a look at the behavior of the integral term mentioned above and conclude that it indeed becomes substantial for distances comparable to the wire radius.

\section{ACKNOWLEDGMENTS}

D.D. acknowledges financial support by the OPTIMAS Carl-Zeiss-Ph.D. program and by the Research Fund of the Hungarian Academy of Sciences (OTKA) under Contract No. NN 78112.

\section{APPENDIX A: KRAMERS-KRONIG RELATION GENERALLY}

First, let us look at the Kramers-Kronig relations in the case of a general, complex-valued function $f(\omega)$ that is analytic in the upper complex half plane. According to Cauchy's theorem,

$$
f\left(\omega_{A}\right)=\lim _{\delta \rightarrow 0^{+}} \frac{1}{2 \pi i} \oint_{C} d \omega \frac{f(\omega)}{\omega-\omega_{A}-i \delta},
$$

and the contour (containing the point $\omega_{A}+i \delta$ within) is closed in the upper complex half-plane. If we assume that the path integral of $f(\omega)$ is nonzero only over the real axis and disappears on the other parts of the contour (that is, on the complex plane) if we extend it to infinity, we can write the integral as

$$
f\left(\omega_{A}\right)=\lim _{\delta \rightarrow 0^{+}} \frac{1}{2 \pi i} \int_{-\infty}^{\infty} d \omega \frac{f(\omega)}{\omega-\omega_{A}-i \delta} .
$$

Since

$$
\begin{gathered}
\frac{1}{\omega-\omega_{A}-i \delta}=\mathbb{P}\left(\frac{1}{\omega-\omega_{A}}\right)+i \pi \delta\left(\omega-\omega_{A}\right), \\
f\left(\omega_{A}\right)=\frac{1}{2 \pi i} \mathbb{P} \int_{-\infty}^{\infty} d \omega \frac{f(\omega)}{\omega-\omega_{A}}+\frac{1}{2} f\left(\omega_{A}\right),
\end{gathered}
$$

and so

$$
f\left(\omega_{A}\right)=\frac{1}{\pi i} \mathbb{P} \int_{-\infty}^{\infty} d \omega \frac{f(\omega)}{\omega-\omega_{A}}
$$

or, with real and imaginary parts,

$$
\begin{aligned}
\operatorname{Re}\left\{f\left(\omega_{A}\right)\right\} & =\frac{1}{\pi} \mathbb{P} \int_{-\infty}^{\infty} d \omega \frac{\operatorname{Im}\{f(\omega)\}}{\omega-\omega_{A}}, \\
\operatorname{Im}\left\{f\left(\omega_{A}\right)\right\} & =-\frac{1}{\pi} \mathbb{P} \int_{-\infty}^{\infty} d \omega \frac{\operatorname{Re}\{f(\omega)\}}{\omega-\omega_{A}} .
\end{aligned}
$$

\section{APPENDIX B: ANALYTIC APPROXIMATION OF THE DIPOLE-DIPOLE SHIFT FOR TWO EMITTERS COUPLED BY A NANOWIRE}

The Green's tensor for an infinitely long, cylindrical wire can be calculated as given in Ref. 6. For the scattered part, we can formally write

$$
\overline{\bar{G}}^{\text {med }}\left(\vec{r}_{1}, \vec{r}_{2}, \omega\right)=\int_{-\infty}^{\infty} d k_{z} \tilde{\bar{G}}^{\text {med }}\left(\vec{r}_{1}, \vec{r}_{2}, \omega ; k_{z}\right),
$$


where we know $\tilde{\bar{G}}^{\text {med }}$ analytically. The atom-wire coupling is strongest if the dipole moment of the emitters point in the radial direction. If the cylindrical coordinates of two emitters only differ in their $z$ component (where $z$ is the symmetry axis of the wire), one finds

$$
G_{r r}^{\mathrm{med}}\left(\vec{r}_{1}, \vec{r}_{2}, \omega\right)=\int_{-\infty}^{\infty} d k_{z} e^{i k_{z} \Delta z} \tilde{G}_{r r}^{\mathrm{med}}\left(\vec{r}_{1}, \vec{r}_{1}, \omega ; k_{z}\right),
$$

where $\Delta z=\left|z_{2}-z_{1}\right|$. In case of a thin wire (radius well below the vacuum radiation wavelength of the emitter), and small emitter-wire distance (in the order of magnitude of the radius), the plasmonic contribution becomes dominant in $\tilde{G}_{r r}^{\text {med }}\left(\vec{r}_{1}, \vec{r}_{1}, \omega ; k_{z}\right)$. In this case, the imaginary part of the Green's tensor is well approximated by two Lorentzian fits, centered at $\pm k_{z}^{\mathrm{pl}}$, i.e., the $z$ component of the wave vector of the plasmonic mode. This approximation is valid for interemitter distances larger than the vacuum radiation wavelength because, in this case, the only substantial channel that couples the emitters are the surface plasmon modes:

$$
\operatorname{Im}\left\{\tilde{G}_{r r}^{\operatorname{med}}\left(\vec{r}_{1}, \vec{r}_{1}, \omega ; k_{z}\right)\right\} \approx \frac{A(\omega)}{1+\frac{\left[k_{z}-k_{z}^{\mathrm{p}}(\omega)\right]^{2}}{\gamma(\omega)^{2}}}+\frac{A(\omega)}{1+\frac{\left[k_{z}+k_{z}^{\mathrm{p}}(\omega)\right]^{2}}{\gamma(\omega)^{2}}} .
$$

Because of the translational invariance of the wire in the $z$ direction, $\tilde{\overline{\bar{G}}}^{\text {med }}$ is symmetric in $k_{z}$. Thus, substituting in Eq. (B2), we get

$$
\operatorname{Im}\left\{G_{r r}^{\mathrm{med}}\left(\vec{r}_{1}, \vec{r}_{2}, \omega\right)\right\}=\operatorname{Im}\left\{2 \pi i A \gamma e^{i k_{z}^{\mathrm{pl}} \Delta z} e^{-\gamma \Delta z}\right\} .
$$

With this, we can now express the wire-induced singleemitter decay rate and emitter-emitter coupling, respectively,

$$
\begin{gathered}
\Gamma_{11}^{\mathrm{appr}}=\frac{4 d^{2} \pi \omega_{A}^{2}}{\hbar \epsilon_{0} c^{2}} A\left(\omega_{A}\right) \gamma\left(\omega_{A}\right), \\
\Gamma_{12}^{\mathrm{appr}}=\frac{4 d^{2} \pi \omega_{A}^{2}}{\hbar \epsilon_{0} c^{2}} A\left(\omega_{A}\right) \gamma\left(\omega_{A}\right) e^{-\gamma \Delta z} \cos \left(k_{z}^{\mathrm{pl}} \Delta z\right),
\end{gathered}
$$

as well as the wire-induced dipole-dipole shift, according to Eq. (18):

$$
\begin{aligned}
\delta \omega_{12}^{\mathrm{appr}}= & -\frac{2 \pi d^{2} \omega_{A}^{2}}{\hbar \epsilon_{0} c^{2}} A\left(\omega_{A}\right) \gamma\left(\omega_{A}\right) e^{-\gamma \Delta z} \sin \left(k_{z}^{\mathrm{pl}} \Delta z\right) \\
& +\frac{d^{2} \omega_{A}}{\hbar \epsilon_{0} \pi} \int_{0}^{\infty} d \kappa \frac{\kappa^{2}}{c^{2}} \frac{\operatorname{Re}\left\{2 \pi i A \gamma e^{-\gamma \Delta z} e^{i k_{z}^{\mathrm{p}} \Delta z}\right\}_{\omega=i \kappa}}{\kappa^{2}+\omega_{A}^{2}} .
\end{aligned}
$$

According to the discussion in the paper, for interemitter distances larger than the vacuum radiation wavelength, the integral term in Eq. (B7) can be neglected, so in the end we arrive at the analytic approximation:

$$
\delta \omega_{12}^{\mathrm{appr}} \approx-\frac{2 \pi d^{2} \omega_{A}^{2}}{\hbar \epsilon_{0} c^{2}} A\left(\omega_{A}\right) \gamma\left(\omega_{A}\right) e^{-\gamma \Delta z} \sin \left(k_{z}^{\mathrm{pl}} \Delta z\right) .
$$

*Now at German Aerospace Center (DLR), Institute of Technical Physics, Stuttgart, Germany.

${ }^{1}$ S. Y. Buhmann, H. T. Dung, and D.-G. Welsch, J. Opt. B: Quantum Semiclass. Opt. 6, S127 (2004).

${ }^{2}$ S. Y. Buhmann, L. Knöll, D.-G. Welsch, and H. T. Dung, Phys. Rev. A 70, 052117 (2004)

${ }^{3}$ S. Y. Buhmann and D.-G. Welsch, Prog. Quant. El. Dyn. 31, 51 (2007).

${ }^{4}$ D. E. Chang, A. S. Sørensen, P. R. Hemmer, and M. D. Lukin, Phys. Rev. Lett. 97, 053002 (2006).

${ }^{5}$ D. E. Chang, A. S. Sørensen, P. R. Hemmer, and M. D. Lukin, Phys. Rev. B 76, 035420 (2007).

${ }^{6}$ D. Dzsotjan, A. S. Sørensen, and M. Fleischhauer, Phys. Rev. B 82, 075427 (2010).

${ }^{7}$ Y. Chen, N. Gregersen, T. R. Nielsen, J. Mørk, and P. Lodahl, Opt. Express 18, 12489 (2010).
${ }^{8}$ D. Martin-Cano, L. Martin-Moreno, F. J. Garcia-Vidal, and E. Moreno, Nano Lett. 10, 3129 (2010).

${ }^{9}$ D. E. Chang, A. S. Sørensen, E. A. Demler, and M. D. Lukin, Nat. Phys. 3, 807 (2007).

${ }^{10}$ A. V. Akimov, A. Mukherjee, C. L. Yu, D. E. Chang, A. S. Zibrov, P. R. Hemmer, H. Park, and M. D. Lukin, Nature (London) 450, 402 (2007).

${ }^{11}$ J. Kästel and M. Fleischhauer, Phys. Rev. A 71, 011804(R) (2005).

${ }^{12}$ P. de Vries, D. V. van Coevorden, and A. Lagendijk, Rev. Mod. Phys. 70, 447 (1998).

${ }^{13}$ D. E. Chang, Jun Ye, and M. D. Lukin, Phys. Rev. A 69, 023810 (2004).

${ }^{14}$ A. Gonzalez-Tudela, D. Martin-Cano, E. Moreno, L. MartinMoreno, C. Tejedor, and F. J. Garcia-Vidal, Phys. Rev. Lett. 106, 020501 (2011)

${ }^{15}$ C. P. Van Vlack, P. Yao, and S. Hughes, e-print arXiv:1102.0794. 